\title{
Conductas autolíticas en los adolescentes. Estudio realizado a dos colectivos étnicos
}

\author{
Dinesh Bhugra* Dr, Senior Lecturer \\ Jayshree Singh** Dr, Investigador \\ E. Fellow-Smith ${ }^{\star \star \star}$ Dr, Psiquiatría Infanto- \\ juvenil \\ Carol Bayliss ${ }^{\star \star \star \star}$ Dr, Asistente Clínico \\ * Instituto de Psiquiatría De Crespigny Park \\ London SE5 8AF \\ ** Windmill Lodge West London NHS \\ Healthcare Trust Uxbridge Road Southall \\ Middx UB1 3EU \\ *** West London NHS Healthcare Trust \\ Southall Middx UB1 3EU \\ **** West London NHS Healthcare Trust \\ Southall Middx UB1 3EU \\ REINO UNIDO
}

\begin{abstract}
RESUMEN - Antecedentes: Estudios previos indican que los adolescentes asiáticos no presentan una tendencia más acusada a la conductas autolíticas que los adolescentes de raza blanca a pesar de la existencia de diferentes factores sociales que contribuyen a este tipo de conductas.

Método: Durante un periodo de más de tres años se realizó una recogida y clasificación de los casos de personas de origen asiático y de los de raza blanca que habían sido evaluados por la Unidad Infanto-juvenil por haber realizado una conducta autolítica. Obtuvimos información a cerca del intento de suicidio así como de las circunstancias que lo rodearon.

Resultados: Identificamos un total de 99 casos de los cuales 76 fueron protagonizados por personas de raza blanca y 23 por personas de razas asiáticas. Un número significativo de los adolescentes en ambos grupos tenían 14 años de edad o más y las chicas presentaban una tendencia más acusada al suicidio que los chicos. En 43 casos el intento de suicidio fue el resultado de un impulso (35 personas de raza blanca y 8 de razas asiáticas) y 29 casos (el 30\%) tenían antecedentes de conducta autolítica. El método más utilizado fue el la sobredosis ( 90 casos, 67 blancos y 23 asiáticos). El paracetamol, solo o combinado con otras, fue el fármaco más utilizado (en 44 casos, 35 personas de raza blanca y 9 de razas asiáticas). En 32 casos, (25 personas de raza blanca y 7 de razas asiáticas), las discusiones con los padres o hermanos fueron el factor precipitante, por otro lado, 18 adolescentes afirmaron tener problemas en la escuela. Asimismo, 16 chicos reconocieron que mantener actitudes amenazadoras o desafiantes en clase fue la razón por la que llegaron a ingerir una sobredosis. 22 chicos de raza blanca (el 29\%) y 10 asiáticos (el 43\%) adujeron el abuso sexual como motivo principal.
\end{abstract}


Conclusiones: Los adolescentes asiáticos no presentan tendencias más acusadas a tomar sobredosis que el resto de los jóvenes, sin embargo la influencia que el abuso sexual tiene en estas actitudes requiere un estudio en profundidad.

\section{Introducción}

La proporción de conductas autolíticas ha aumentado durante los últimos años, y ha sido catalogada como epidemia. (Hawton 1986). Existen evidencias clínicas que sugieren que tanto los niños como los adolescentes que han intentado suicidarse suelen tener antecedentes familiares de alcoholismo y un comportamiento más agresivo (Cohen-Sandler 1982). Estudios anteriores sugieren que los problemas familares, especialmente los que se tienen con los padres, novio o novia así como los conflictos en la escuela son factores precipitantes a la hora de provocar un intento de suicidio entre los adolescentes (Hawton et al. 1982a). Normalmente, los chicos aducen sentimientos de soledad, de ira o furia y afirman no sentirse queridos. Se ha dicho que el intento de suicidio es en realidad una forma de manifestar o aliviar este malestar (Hawton et al. 1982b).

Muchos intentos van unidos a dificultades familiares existentes y se ha señalado que previamente los adolescentes habían acudido a los servicios sociales o a la Seguridad Social con asiduidad (Clarke 1988). Normalmente, los adolescentes afirman que sus padres muestran una actitud fría, no comunicativa y son incapaces de tolerar o aceptar cambios o crisis. Sus hijos los perciben como personas hostiles o indiferentes (Garfinkel et al. 1982, Taylor \& Stansfield 1984). Hawton et al. (1982a) afirmaron que el $80 \%$ de los chicos que habían intentado suicidarse confesaron su incapacidad para hablar de sus problemas con su padre y, alrededor de la mitad de ellos tenían estas mismas dificultades con su madre.

La cantidad de datos que existen sobre los intentos de suicidio es limitada. Biswas (1990), en un estudio retrospectivo, afirmó que las chicas asiáticas tendían a describir sus intentos de suicidio como accidentales y acudieron a los servicios sociales en menor número. McGibben et al. (1992) señaló que las medias anuales de ingresos hospitalarios eran parecidas entre los adolescentes caucásicos y los asiáticos y en ambas, las chicas superaban a los chicos. En un estudio previo, realizado en la misma ciudad (Coventry), Handy et al. (1991) identificó los conflictos culturales intergeneracionales, como importantes factores precipitantes.

Kingsbury (1994), en un estudio realizado a 50 adolescentes, de los cuales 13 eran asiáticos y el resto de raza blanca, señaló que los asiáticos se aislaban más y, a pesar de sus bajos niveles de intento de suicidio, mostraban índices superiores de depresión, sentimientos de abandono y una mayor premeditación con sobredosis previas. Además, argumentaba que el intento de suicidio no debería ser un elemento de juicio determinante. Según los estudios realizados en Escocia por Lockhart (1998), el 93\% de los casos, los adolescentes habían ingerido sobredosis durante la primera fase de la investigación y el $87 \%$ durante la segunda fase y que las mujeres presentaban una tendencia más acusada a realizar intentos de suicidio. Por otro lado, Nasr et al. (1997) 
descubrieron, a través de los estudios realizados en Birmingham, que los intentos de suicidio se llevaban a cabo mediante sobredosis de fármacos y el 5\% de las personas estudiadas había repetido el intento en un periodo de seguimiento de seis meses. Sin embargo, en este estudio no se realizaba ninguna distinción entre las etnias. Goddard et al. (1996) mostraron que las proporciones entre los adolescentes negros, en lo que respecta a una conducta autolítica,eran proporcionales al tipo o a la clase de miembros que formaban la comunidad. Las variables sociodemográficas entre adolescentes de raza blanca y raza negra eran parecidas, con la excepción de que el primer grupo padecía de un mayor estrés social.

En este estudio hemos utilizado los términos 'conducta autolítica' e 'intento de suicidio' de forma indistinta. Ambos definen cualquier acto que pueda constituirse como un intento por dañarse a sí mismo. Empezamos estudiando las características sociodemográficas de todos los adolescentes que habían acudido a la Unidad Infanto-juvenil teniendo en cuenta la posibilidad de que existieran claras diferencias étnicas. Además, se decidió estudiar algunos factores de riesgo tales como los antecedentes previos, las alteraciones de la conducta, así como el mal empleo que se hace de ciertas sustancias ya que, según varios estudios (Lewinshan et al. 1994; Morrisey et al. 1995), tienen una conexión directa con las conductas autolíticas. clínicas así como la información obtenida a partir de los datos sociodemográficos, incluyendo su raza. Intentamos obtener toda la información disponible en las historias clínicas relativa a las conductas autolíticas, al igual que cualquier otro factor precipitante según la opinión de los médicos encargados de valorar el intento de suicidio.

La etnia a la que pertenecía cada uno de los individuos fue determinada por ellos mismos o por sus padres. Sólo se incluyeron en el estudio dos grupos de personas, los de raza blanca y de razas asiáticas, incluyendo en éste último a aquellos adolescentes cuyos padres habían nacido o procedían del subcontinente indio.

Utilizamos un formato estándar con el fin de saber cuáles eran los factores precipitantes que, a su vez, fueron divididos en amplios aspectos tales como la de conflictos con la familia, la dificultad en las relaciones sociales, los problemas en la escuela, un ambiente poco favorable en casa y otros problemas como el de evitar un Juicio legal contra él. Además, se recogieron datos sobre la relación con los padres y antecedentes de abusos físicos y/o sexuales.

Identificamos aquellos factores predisponentes al suicidio, especialmente en lo que se refiere a su relación con los compañeros y la familia.

\section{Resultados}

\section{Método}

Todos los casos de conductas autolíticas que fueron presentados ante el equipo de evaluación infanto-juvenil, se estudiaron durante un periodo de tiempo de tres años. También se tuvieron en cuenta las historias
Identificamos 99 casos de intento de suicidio y estudiamos sus historias clínicas. De ellos, 76 (el 77\%) correspondían a personas de raza blanca y 23 (el 23\%) a asiáticos. De las 76 personas de raza blanca, 13 (el 17\%) eran hombres y el resto mujeres (M:F ratio de $1: 4,8)$. 
Tabla I

Distribución de las edades en dos grupos (\% entre paréntesis)

\begin{tabular}{lcc} 
& Blancos & Asiáticos \\
& $\mathrm{n}=76$ & 23 \\
\hline$<10$ años & $2(2,6)$ & 0 \\
$11-13$ años & $19(25)$ & $7(30)$ \\
$14-15$ años & $52(68)$ & $13(56)$ \\
$>15$ años & $3(4)$ & $3(13)$ \\
\hline
\end{tabular}

En lo que respecta al grupo de asiáticos, 2 (el $0,8 \%$ ) eran hombres y el resto mujeres (M:F ratio de 1:10,5). La distribución de edad entre los dos grupos aparece en la tabla I.

Los servicios locales se ocupan de los adolescentes sólo hasta la edad de 16 años. Los datos se presentan según la etnia y no el sexo.

El origen de la conducta autolítica fue de carácter impulsivo en 35 adolescentes de raza blanca (el $46 \%$ ) y premeditado en 10 (el 13\%), en el resto, el motivo no quedó claro. En cuanto a los asiáticos, el intento fue impulsivo en 8 casos (el 35\%) y premeditado en 4 (el 1,7\%), no existiendo información disponible en lo que al resto se refiere. De un total de 76 adolescentes de raza blanca, 23 (el 30\%) tenían un historial previo de conducta autolítica, mientras que en el caso de los asiáticos se trataba de 6 (el $26 \%$ ). Tan sólo 2 personas de raza blanca habían preparado una nota de suicidio. Por otro lado, 14 adolescentes de raza blanca (el $18 \%$ ) y 2 asiáticos (el 8\%) admitieron que su intento de suicidio buscaba llamar la atención de alguien. 16 personas de raza blanca (el 21\%) y 6 de razas asiáticas (el $26 \%$ ) querían morir frente a 8 adolescentes de raza blanca (el 10\%) y 6 asiáticos (el $26 \%$ ) que no lo deseaban y no se dispuso de esta información en el resto de los casos. 16 chicos de raza blanca (el 21\%) y 5 asiáticos (el 22\%) intentaron evitar que se supiera. 21 blancos (el $27 \%$ ) y 5 de raza asiática se arre- pintieron de haberlo intentado. 18 blancos (el 23\%) acudieron a los servicios sociales y otros 2 fueron adoptados, en el caso de los asiáticos, la cifra fue de 5 (el 22\%).

El método más común fue la sobredosis, en 67 personas (el 88\%), en lo que al grupo de personas de raza blanca se refiere, 5 jóvenes (el 6\%) lo intentaron cortándose, otros 3 (el $4 \%$ ) mediante ahorcamiento o estrangulamiento y una persona bebió lejía. En cuanto a los asiáticos, todos los individuos ingirieron una sobredosis. La droga más utilizada fue el paracetamol, ya fuera ésta sola (22 personas de raza blanca y 6 asiáticos) o combinada con otras (13 y 3 casos respectivamente). El resto de los adolescentes que tomó sobredosis realizó otras combinaciones.

Los factores precipitantes aparecen en la tabla II según los describieron los mismos adolescentes o sus tutores. Estos son los elementos que precipitaron el intento de suicidio y que han de ser diferenciados de otro tipo de tensiones.

11 personas de raza blanca (el 14\%) y 5 asiáticos (el 22\%) reconocieron haber tenido problemas en la escuela.

En el grupo de adolescentes de raza blanca, los padres estaban separados en 38 casos frente a un solo caso en el grupo de los asiáticos. Únicamente 12 padres de raza blanca estaban casados y 14 convivían con otra pareja después de haberse separado. 8 adolescentes de raza blanca (el 10\%) y 3 asiáti- 
Tabla II

Factores precipitantes (el total exede del número por múltiples problemas)

\begin{tabular}{lcc} 
& $\begin{array}{c}\text { Blancos } \\
\mathrm{n}=76\end{array}$ & $\begin{array}{c}\text { Asiáticos } \\
23\end{array}$ \\
\hline Argumentos & $25(32)$ & $7(30)$ \\
$\quad$ Familia & $2(3)$ & $1(4)$ \\
$\quad$ Amigos & $1(1,3)$ & 0 \\
$\quad$ Otros & $4(5)$ & \\
Problemas de relación & $2(3)$ & \\
$\quad$ Con los padres & $8(10)$ & \\
$\quad$ Con los iguales & $8(10)$ & $1(4)$ \\
$\quad$ Novios & 0 & $4(17)$ \\
Ambiente hogareño desfavorable & $40(53)$ & $7(30)$ \\
Conflicto cultural & $3(4)$ & 0 \\
Problemas escolares & & \\
Problemas legales &
\end{tabular}

$\cos$ ( el 13\%) afirmaron haber sufrido abusos físicos y 14 blancos (el 18\%) y 7 asiáticos (el 30\%) abusos sexuales. En 18 casos (14 adolescentes de raza blanca, el $18 \%$ y 4 de razas asiáticas, el $2 \%$ ) existían trastornos de comportamiento. Un total de 6 personas ( 3 en cada grupo) admitieron haber consumido sustancias ilegales.
Los factores que predisponen al suicidio están recogidos en la tabla III y son los que se han mantenido constantes a lo largo del tiempo.

Estos datos muestran que un ambiente poco favorable en el hogar es un factor importante. Sin embargo, no todos los casos estudiados permitieron identificar a los fac-

Tabla III

Factores predisponentes

\begin{tabular}{lcc} 
& $\begin{array}{c}\text { Blancos } \\
\mathrm{n}=76\end{array}$ & $\begin{array}{c}\text { Asiáticos } \\
23\end{array}$ \\
\hline Relaciones & & \\
$\quad$ Padres & $15(20)$ & $1(4)$ \\
Hermanos & $5(6)$ & 0 \\
Otros & $1(1,3)$ & $1(4)$ \\
Ambiente hogareño desfavorable & $5(6)$ & 0 \\
$\quad$ Problemas parentales & $4(5)$ & $1(4)$ \\
Violencia & $5(6)$ & 0 \\
Enf. mental parental & $6(8)$ & $1(4)$ \\
Abuso físico & $6(8)$ & $6(23)$ \\
Abuso sexual & $6(8)$ & $2(8)$ \\
Alcoholismo parental & $2(2,5)$ & $10(43)$ \\
Conflicto cultural & & \\
Escuela & $6(8)$ & $8(35)$ \\
$\quad$ Problemas generales & $1(1,3)$ & $3(13)$ \\
Comportamientos "chulescos" & & \\
\hline
\end{tabular}


tores predisponentes razón por la cual las cifras no siempre coinciden.

\section{Discusión}

Para interpretar los resultados de este estudio, es necesario tener ciertos elementos en cuenta. En primer lugar, se trata de un estudio retrospectivo de historias clínicas y, como ocurre con todos los estudios de este tipo, existen limitaciones en cuanto a la cantidad y la calidad de los datos disponibles. En segundo lugar, las cifras aún son pocas, especialmente en lo que respecta a los grupos asiáticos, por lo que resulta difícil establecer generalidades. En tercer lugar, también habrá que tener en cuenta la heterogeneidad de ambos grupos a la hora de interpretar los datos.

La distribución por edad de la muestra confirma los hallazgos de otros autores. El marcado aumento a la edad de 14 años, no hace sino mostrarnos el impacto que supone la pubertad. Es también posible que ésta sea la edad en la que comienza a desarrollarse el individualismo y a crearse tensiones en casa del mismo modo, también las inhibiciones comienzan a jugar un papel importante, aunque este hecho tendrá que ser estudiado en trabajos posteriores.

\section{Naturaleza del intento de suicidio}

En toda la muestra, el método más común fue el de la sobredosis -tan sólo un reducido número de ellos lo intentó cortándose las venas. El hecho de que la gran mayoría de la muestra, en ambos grupos, tomase paracetamol, ya fuera sólo o combinado, confirma tanto los descubrimientos de Lockhart (1998) como los de Nasr et al. (1997). Parece lógico pensar que cada persona utilice los medios a su alcance sobre todo si se trata de un acto impulsivo. De este modo, los analgésicos se convierten en los primeros candidatos en caso de sobredosis, por lo que cualquier estrategia que se utilice de modo preventivo tendrá que incluir una limitación del acceso a estos productos y la adición de compuestos como metionina al paracetamol con el fin de reducir su toxicidad.

\section{Ambiente desfavorable en casa}

Las diferencias de sexo no son sorprendentes, sin embargo el alto número de separación entre los padres, con especial incidencia en los grupos de raza blanca resultó ser un descubrimiento extraordinario. En algunos casos, el abuso de alcohol por parte de uno de los padres o hermanos resultó ser un factor importante. El abuso sexual o físico en casa de forma repetida junto a los problemas en el colegio y la escasa relación con los compañeros parecen contribuir a aumentar las presiones a las que estos individuos se sienten sometidos. El hecho de ingerir una sobredosis les da 'tiempo muerto', aunque sólo por un momento. Dos individuos afirmaron haber sido discriminados por su raza. En uno de los casos, una adolescente asiática no se lo contó a sus padres porque quería 'protegerlos'. En el segundo, el chico sentía que las clases altas abusaban de su madre quien trabajaba como empleada del hogar y sentía además, que todo este colectivo estaba siendo explotado.

Se pueden ofrecer dos posibles argumentos para explicar las diferencias sexuales que existen entre los asiáticos, mucho más marcadas que entre los adolescentes de raza blanca. En primer lugar, las expectativas que la familia y la comunidad tienen según el sexo son muy diferentes cuando se compara 
a los chicos asiáticos con las chicas del mismo origen. Éstas últimas pueden ser consideradas como una carga familiar y la actitud de la familia ante su comportamiento suele ser más estricta. Las chicas deben comportarse de un modo más tradicional, compartir las obligaciones que implican las tareas del hogar y, en el colegio, aún se espera que actúen igual que el resto de sus compañeros. Este conflicto, junto al abuso, las escasas relaciones interpersonales así como los conflictos familiares pueden dar lugar a una situación insoportable para ellas y hacer que las sobredosis se conviertan en una fórmula de escape. En segundo lugar, si pasan tiempo en casa evitando así ir a la escuela, tendrán mayores posibilidades de presenciar los conflictos de pareja entre sus padres. Sin embargo, resulta difícil determinar tanto los conflictos matrimoniales como los culturales a través de datos retrospectivos, por lo que se han preparado estudios prospectivos con el fin de tratar estos temas.

\section{Problemas en la escuela}

Existen dos aspectos fundamentales en los que se espera que un adolescente responda, por ello no es sorprendente que los problemas en la escuela afecten tanto a la precipitación como a la predisposición al intento de suicidio. Más de la mitad de las personas de raza blanca y un tercio de las de raza asiática afirmaron que los problemas en clase fueron las factores precipitantes, mientras que el $9 \%$ de los blancos y el $48 \%$ de los asiáticos lo citaron como un elemento que predisponía a ello. De cualquier modo, los problemas en el colegio (entre los que se incluyen las dificultades académicas, problemas con los compañeros etc.) pueden contribuir a crear una sensación de abandono y desamparo, lo que tendrá que ser estudiado en trabajos posteriores. Estas dificul- tades, unidas a los problemas en casa, indican que puede que los adolescentes vulnerables no tengan otra fómula de escape que la de tomar una sobredosis y conseguir un 'tiempo muerto'. Nasr et al. (1997) descubrieron que las discusiones familiares así como aquellos factores estresantes relacionados con el colegio estaban relacionados en el $64 \%$ de los casos y los descubrimientos realizados en este estudio lo confirman. McGibben et al. (1992) habían observado que se producían más ingresos hospitalarios durante el periodo escolar que durante las vacaciones, lo que implica que la escuela puede actuar como un factor estresante.

Los descubrimientos relacionados con la raza sugieren, al igual que los aportados por Goddard et al. (1996) que la etnia en sí misma no es un elemento determinante de los índices de conductas autolíticas en adolescentes como tampoco lo es de los intentos de suicidio (Kingsbury 1994). Es imposible calcular las tasas de incidencia a partir de los datos aportados por las historias clínicas.

El sentimiento general que se desprende de estos casos es el de la desesperación ante la propia realidad a la que se enfrentan estos adolescentes y la que les presentan sus familias, así como la sensación de sentirse abandonados por sus seres queridos y también en la escuela. Se necesitan estudios descriptivos para lograr saber cuáles fueron las causas de sobredosis y el intento en sí mismo, con el fin de establecer estrategias preventivas. De nuestro estudio se puede deducir que las escuelas, los padres y los profesionales de la salud han de trabajar juntos con el fin de determinar estas causas. Aquellos elementos que influyen en la repetición de las sobredosis, una vez determinados, pueden ser utilizados con el fin de reducir los intentos de suicidio y para que los jóvenes puedan optar por otras formas de expresar su angustia que no sea una potencialmente fatales para ellos 
mismos. Las diferencias según la etnia no están determinadas, sin embargo es necesario subrayar las tensiones familiares, el abuso sexual y físico, así como los problemas en la escuela, todo ello con el fin de plantear las actuaciones apropiadas.

\section{Bibliografía}

BISWAS, S. Ethnic differences in self-poisoning: a comparative study between Asian and white adolescent groups. Journal of Adolescence 13: 189-193, 1990.

CLARKE, C.F. Deliberate self-poisoning in adolescents. Arch Dis Child 63: 1479-1483, 1988.

COHEN-SANDLER, R., BERMAN, A., KING, R. A follow-up study of hospitalised suicidal children. Journal of the American Academy of Child Psychiatry 21:398-403, 1982.

GARFINKEL, B., FROESE, A., HOOD, J. Suicide attempts in children and adolescents. American Journal of Psychiatry 139: 1257-1261, 1982.

GODDARD, N., SUBOTSKY, F., FOMBONNE, E. Ethnicity and adolescent deliberate self-harm. Journal of Adolescence 19: 513-527, 1996.

HAWTON, K. Suicide and attempted suicide among children and adolescents, in Developmental Clinical Psy chology and Psychiatry London: Sage, 1986.

HAWTON, K, O'GRADY, J.,OSBORN, M.,COLE, D. Adolescents who take overdoses British Journal of Psy chiatry 140: 118-1231, 1982a.
HAWTON, K., COLE, D., O'GRADY, J., OSBORN, M. Motivational aspects of deliberate self poisoning in adolescents. British Journal of Psychiatry 141: 286-291, $1992 b$.

KINGSBURY, S. The psychological and social characteristics of Asian adolescent overdose Journal of Adoles cence 17: 131-135, 1994.

LEWISHAN, R., ROHDE, P.,SEELEY, J. Psychosocial risk factors for future suicide attempts. Journal of Consul ting and Clinical Psychology 62: 297-305, 1994.

MORRISEY, R., DICKEN, R., ABIKOFF, H. et al. Hospitalising the suicidal adolescent:investigation of decision-making criteria. Journal of the American Academy of Child and Adolescent Psychiatry 34: 902-911, 1995.

TAYLOR, E., STANSFIELD, S. Children who poison themselves. British Journal of Psychiatry 145:127-135,1984.

HANDY, S.,CHITHIRANDIAN, R., BALLARD, C. et al. Ethnic differences in adolescent self poisoning: a comparison of Asian and Caucasian groups. Journal of Adoles cence 14: 157-162, 1991.

McGIBBEN, L., BALLARD, C.,HANDY, S.,MOHAN, R., SILVEIRA, W. Deliberate self-poisoning in Asian and Caucasian 12-15 years old. British Journal of Psychiatry 161: 110-112, 1992.

Dirección para correspondencia:

Dr. Dinesh Bhugra

Institute of Psychiatry

De Crespigny Park

London SE5 8AF

UNITED KINGDOM 